Ann. Nucl. Energy Vol. 23, No. 8, pp. 625-640, 1996

Copyright (C) 1996 Elsevier Science Ltd

\title{
CONFLUENCE OF CALCULATIONAL AND EXPERIMENTAL INFORMATION FOR DETERMINATION OF POWER DISTRIBUTION AND BURNUP
}

\author{
I. V. SEROV AND J. E. HOOGENBOOM \\ Delft University of Technology, Interfaculty Reactor Institute, Mekelweg 15, 2629 JB Delft, \\ The Netherlands
}

(Received 6 April 1995)

\begin{abstract}
A technique for the statistical confluence of any number of possibly correlated informational sources employed in reactor analysis can be used to improve the estimates of physical quantities given by the sources taken separately. The formulas of the presented technique being based on multivariate Bayesian conditioning are general and can be employed in different applications. Insight into the nature of the informational source allows different types of data associated with the source to be improved. Estimation of biases, variances and correlation coefficients for the systematic and statistical errors associated with the informational sources is essential for reliable confluence, but pays off by providing optimal estimates. The technique of the calculational and experimental information confluence is applied to the determination of the power distribution and burnup for the research reactor HOR of the Delft University of Technology. The code system CONHOR carries out all the stages of the calculation for the HOR reactor, using an existing code for static core calculations and burnup calculations.
\end{abstract}

\section{INTRODUCTION}

Any informational source employed in nuclear reactor analysis is associated with errors. Two or more advanced and accurate codes will always deliver different estimates for the same set of physical quantities. An experiment can serve as an instrument of additional judgement. Generally, the informational sources can be correlated with each other if, for example, similar models or data are used. The sources bearing different, but correlated, quantities can be used for mutual improvement if the degree of the correlation, carrying the additional knowledge, is known. Mutual correlation between the quantities is a natural phenomenon in reactor core analysis. Therefore, the mutual correlation between these quantities carries additional knowledge as well and the multi-variate Bayesian conditioning (Lee, 1989) is naturally fruitful for seeking their best estimates. In these circumstances it is imperative to have a formal mathematical instrument, allowing us to employ this seemingly redundant knowledge in order to pick up the best estimates. The formulas for the general multi-source, multi-variate statistical confluence of the correlated information are derived and presented in the following section. In contrast to the intuitive and intricate derivation of the general adjustment prescriptions given by Perel et al. (1994), we exploit the Bayesian statistics, taking advantage of its fundamental significance for any type of data adjustment or evaluation, confirmed again by Fröhner (1994). The downto-earth problem is how to estimate the prior knowledge required for the confluence, namely statistical properties of the uncertainties (both statistical and systematic). This problem is far from being cleared up in practice, although one of the possible approaches is described in Section 2. We have generally used empirical estimations of these quantities. Following the recommendations, given by Fröhner (1994), we allow simultaneous Gaussian treatment of the systematic and statistical uncertainties. The basic formulas of the multi-variate Bayesian conditioning are cited in the Appendix.

Confluence of two independent sources - calculation and experiment-is a specific case of the general confluence. This is the formalism, which we practiced in our application. The corresponding formulas are secured in Section 3. Distinction is made between uncertainties originated by the calculational model itself 
and the ones linearly propagated from the parameters, allowing for separate model and parameter adjustments. Techniques equivalent to the latter one (confluence of independent calculation and experiment) were tested recently by Bryson et al. (1993) on flux mapping optimization. They give a beneficial survey of the necessity of utilization and the former state-of-the-art of core mapping schemes. We attempted to apply the formalism to improve the accuracy of the determination of the power distribution and burnup of the HOR research reactor. An integrated code system CONHOR is developed to match the CITATION (Fowler et al., 1971) results with the in-core experimental data through confluence. The questions related to the application are described in Section 4. The analysis of the results is presented in Section 5.

\section{A GENERAL TECHNIQUE FOR CONFLUENCE OF DIFFERENT INFORMATIONAL SOURCES}

We consider $K$ different methods of determination of the same data set, consisting of $M$ entries. The methods are subject to both systematic and statistical uncertainties, denoted correspondingly by the vectors $\mathbf{b}_{k}$ and $\mathbf{r}_{k}, k=1, \ldots, K$. Systematic uncertainties originate from common errors of the models and parameters employed in the informational flow of the source and describe correlation between the uncertainty exhibition, characterizing the source. For example, if a single and not absolutely accurate physical model of a control rod is utilized in a core mapping calculation for different reactor states, these states will be correlated with each other and a systematic bias in the results of the calculation can be observed. Statistical uncertainties existing besides the systematic ones originate from statistical fluctuations in the model, parameters and numerical manipulations. In systems with limited knowledge the Gaussian distribution has proven to be the best choice for data inference for both systematic and statistical uncertainties (Janes, 1983). The statistical uncertainties $\mathbf{r}_{k}$ and $\mathbf{r}_{i}$ are described by zero means and covariance matrices $\left\langle\mathbf{r}_{k} \mathbf{r}_{i}\right\rangle$, where $T$ denotes transpose and $k, i=1, \ldots, K$. For systems with limited knowledge about the magnitudes of the biases, it is suggested (Perel et al., 1994) that we describe the systematic uncertainty by a Gaussian with zero mean and either diagonal $\left\langle\mathbf{b}_{k} \mathbf{b}_{i}^{T}\right\rangle$ (if $k$ equals $i$ ) or zero (if $k$ does not equal $i$ ) covariance matrix, rather than by a rectangular probability distribution. This limited knowledge should be utilized to evaluate variances and not means of the biases, which are in this case assumed to be zero. This preference is based on the Maximum Entropy Principle (Janes, 1983).

Due to the fact that systematic and statistical uncertainties have different origins, there is no correlation between them, so that $\left\langle\mathbf{r}_{k} \mathbf{b}_{i}^{\top}\right\rangle$ is zero. Therefore, they can be summed up to get the total uncertainty $\mathbf{f}_{k}$ of the $k$-th informational source with a trivial mean

$$
\left\langle\mathbf{f}_{k}\right\rangle=\left\langle\mathbf{r}_{k}\right\rangle+\left\langle\mathbf{b}_{k}\right\rangle=\mathbf{0}
$$

and a covariance matrix:

$$
\left\langle\mathbf{f}_{k} \mathbf{f}_{k}^{\top}\right\rangle=\left\langle\mathbf{r}_{k} \mathbf{r}_{k}^{\top}\right\rangle+\left\langle\mathbf{b}_{k} \mathbf{b}_{k}^{\top}\right\rangle
$$

In order to combine the data from separate sources by confluence there is no need to distinguish between systematic and statistical uncertainties.

Prior probability assignment methods are determined by the type of informational source. For example, prior statistical properties of model uncertainties, defined by Bryson et al. (1993), introduced by a reactor code, can be evaluated by comparing the results of modeling for different reactor states with the data for the same quantities obtained by a Monte-Carlo code, which can be able to evaluate covariances together with the quantities themselves and under the condition of accurate problem description, can guarantee the absence of a systematic bias. The model uncertainty depends strongly and in a nonlinear way on the calculational problem, and the accuracy of the code as an informational unity without reference to the particular problem cannot be characterized by a single covariance matrix of model uncertainties. Therefore, the size of the problem space should be small enough to preserve the possibility of such description. As a corollary the number of necessary Monte-Carlo calculations can be significantly reduced if the size of the problem space is cut down so that it does not exceed the range of feasible reactor states. Naturally, to observe only the model differences the simulated input parameters should be the same for a given reactor state, processed by both codes.

The average $\langle Z\rangle$ of the observed differences can serve as the standard deviation of the Gaussian distribution 
with zero mean for the systematic uncertainty (Fröhner, 1994) of the model if the Monte-Carlo modeling is unbiased. The covariance matrix $\left\langle\mathbf{b}_{\mathrm{c}} \mathbf{b}_{\mathrm{c}}\right\rangle=\operatorname{diag}\{\langle\mathbf{Z}\rangle\langle\mathbf{Z}\rangle \mathrm{T}\}$ of the systematic uncertainty of the calculational model is diagonal according to this approach. The observed correlations are then related to the statistical uncertainties and their covariance matrix can be evaluated as follows:

$$
\left\langle\mathbf{r}_{\mathrm{c}} \mathbf{r}_{\mathrm{c}}^{\mathrm{T}}\right\rangle=\left\langle(\mathbf{Z}-\langle\mathbf{Z}\rangle)\left(\mathbf{Z}^{\mathrm{T}}-\left\langle\mathbf{Z}^{\mathrm{T}}\right\rangle\right)\right\rangle-\left\langle\mathbf{m}_{\mathrm{mc}} \mathbf{m}_{\mathrm{mc}}^{\mathrm{T}}\right\rangle,
$$

where the covariance matrix of the unbiased differences of the two codes $\left\langle(\mathbf{Z}-\langle\mathbf{Z}\rangle)\left(\mathbf{Z}^{\mathrm{T}}-\left\langle\mathbf{Z}^{\mathrm{T}}\right\rangle\right)\right\rangle$ and a covariance matrix of the Monte-Carlo uncertainties $\left\langle\mathbf{m}_{\mathbf{m c}} \mathbf{m}_{\mathrm{mc}}^{\mathbf{r}}\right\rangle$ should be calculated. The total model uncertainty is then described by zero mean and covariance matrix:

$$
\left\langle\mathbf{f}_{\mathbf{c}} \mathbf{f}_{\mathbf{c}}^{\mathrm{T}}\right\rangle=\langle\mathbf{Z}\rangle\left\langle\mathbf{Z}^{\mathrm{T}}\right\rangle+\left\langle(\mathbf{Z}-\langle\mathbf{Z}\rangle)\left(\mathbf{Z}^{\mathrm{T}}-\left\langle\mathbf{Z}^{\mathrm{T}}\right\rangle\right)\right\rangle-\left\langle\mathbf{m}_{\mathrm{mc}} \mathbf{m}_{\mathbf{m c}}^{\mathrm{T}}\right\rangle
$$

Assignment of prior properties to the uncertainties of informational sources other than reactor codes will be different in practice, but can be similar in essence.

We now turn to the essence of the posterior confluence of the information from $K$ sources. The methods $k+1$ and $k$ show a difference due to both systematic and statistical uncertainties, which is expressed by the random vectors:

$$
\mathbf{y}_{k}=\mathbf{f}_{k+1}-\mathbf{f}_{k}, \quad k=1, \ldots, K-1
$$

Let us compose a vector $\mathbf{y}$ from the subvectors $\mathbf{y}_{k}$ for all $k=1, \ldots, K-1$ :

$$
\mathbf{y}=\left(\mathbf{y}_{1}, \mathbf{y}_{2}, \ldots, \mathbf{y}_{K-1}\right)
$$

The correlation matrix $\left\langle\mathbf{f}_{k} \mathbf{y}^{\mathrm{T}}\right\rangle$ of the uncertainties of the $k$-th source and the chain vector $\mathbf{y}$ of differences is to be composed a priori. It is constituted from the matrices $\left\langle\mathbf{f}_{k} \mathbf{f}_{i}\right\rangle$ of the covariances for the uncertainties of the sources $k$ and $i$.

Considering the situation, when after realization of all methods the vector $\mathbf{y}$ becomes known and expressed by the observed value $\mathrm{Y}$, we apply the formulas, equation (A1) and equation (A2), for the expected value and covariance matrix of conditional Gaussian distribution to the vectors $\mathbf{f}_{k}$ and $\mathbf{y}$.

The mathematical expectation of the vector $\mathbf{f}_{k}$ under the condition that the vector $\mathbf{y}$ is known is expressed in the following way:

$$
\left.\left\langle\mathbf{f}_{k}\right\rangle_{0}=\left\langle\mathbf{f}_{k} y^{\mathrm{T}}\right\rangle \mathbf{y} \mathbf{y}^{\mathrm{T}}\right\rangle^{-1} \mathbf{Y}
$$

The posterior covariance matrix for this vector is:

$$
\left\langle\mathbf{f}_{k} \mathbf{f}_{k}^{\mathrm{T}}\right\rangle_{\mathrm{o}}=\left\langle\mathbf{f}_{k} \mathbf{f}_{k}^{\mathrm{T}}\right\rangle-\left\langle\mathbf{f}_{k} \mathbf{y}^{\mathrm{T}}\right\rangle\left\langle\mathbf{y} \mathbf{y}^{\mathrm{T}}\right\rangle^{-1}\left\langle\mathbf{y} \mathbf{f}_{k}^{\mathrm{T}}\right\rangle
$$

Deducting the vector $\left\langle\mathbf{f}_{k}\right\rangle_{0}$ from the vector of results obtained by means of the $k$-th method, we get better estimates of the results than are offered by any of the methods. These values as well as the posterior covariance matrices do not depend on the choice of $k$. The posterior variances-the diagonal elements of the posterior covariance matrix-are smaller than the prior ones. The posterior matrices do not depend on the value of the observed difference $\mathbf{Y}$. It means that the degree of variance reduction due to the confluence can be evaluated yet before the comparison, i.e. a priori. It is not necessary that the methods are independent. It is required only that they are not deterministically dependent, so that $\left\langle\mathbf{y y} \mathbf{T}^{\mathrm{T}}\right\rangle$ is not a singular matrix.

As an illustration we can consider the case of estimates $F_{1}$ and $F_{2}$ of the same quantity obtained from two independent informational sources with variances $\operatorname{var}\left(f_{1}\right)$ and $\operatorname{var}\left(f_{2}\right)$. If $Y$ is an observed difference $F_{1}-F_{2}$ between the estimates, then utilization of the scalar analog of equation (7) leads to an optimal estimate $\langle F\rangle_{0}$, which is equivalent in this simplified case to the well-known estimate of inverse variances:

$$
\langle F\rangle_{\circ}=F_{1}-\left\langle f_{1}\right\rangle_{\mathrm{o}}=F_{2}-\left\langle f_{2}\right\rangle_{\mathrm{o}}
$$




$$
\begin{aligned}
& =F_{1}+\operatorname{var}\left(f_{1}\right)\left[\operatorname{var}\left(f_{1}\right)+\operatorname{var}\left(f_{2}\right)\right]^{-1}\left(F_{1}-F_{2}\right) \\
& =\left[F_{1} / \operatorname{var}\left(f_{1}\right)+F_{2} / \operatorname{var}\left(f_{2}\right)\right]\left[1 / \operatorname{var}\left(f_{1}\right)+1 / \operatorname{var}\left(f_{2}\right)\right]^{-1}
\end{aligned}
$$

\section{CONFLUENCE OF TWO INDEPENDENT INFORMATIONAL SOURCES-CALCULATION AND EXPERIMENT}

Here we consider a situation typical for reactor applications, when independently measured and calculated data for some reactor state are available. The measured data are the in-core foil activity counts. The calculated data is the power distribution, which is the desired result of our analysis. As we have accepted in the previous section, there is no need to distinguish between systematic and statistical uncertainties. Therefore, the uncertainties of the experiment, calculational parameters and model are described by zero means and $a$ priori known nontrivial covariance matrices.

It is assumed that a set of so-called auxiliary quantities can be calculated by means of the utilized computer code using the same parameters as for the calculation of the desired quantities. These auxiliary quantities should be chosen such that by means of some transformation their experimental values can be obtained also from the measured data. The transformation can, in general, employ a physical model, correlated with the physical model of the reactor computer code. The confluence formalism allows such model-to-model correlation. In practical applications, however, the magnitude of the correlation can hardly be determined. Therefore, it is preferable to stay as close as possible to the measured data, applying the most simple transformational model trying to avoid correlation with the calculation. For example, flux or power distributions, which are usually desired in the analysis, are not recommended to be utilized as auxiliary quantities in order to avoid correlation between the transformational and the calculational models caused by the correlation between cross sections used in both models. In our application the axially averaged foil activation rates are employed as the auxiliary quantities, which requires only a simple transformation of the foil counts to correct for decay, etc.

We denote $\mathbf{f}_{c}$ as the vector of uncertainties of the calculation of the data vector, which consists of two subvectors $f_{a}$ and $f_{d}$, containing the uncertainties of the auxiliary and desired quantities, respectively. They can be detached by means of a projection operation (in every row of the matrices $\mathbf{P}_{\mathrm{a}}$ and $\mathbf{P}_{\mathrm{d}}$ there is one unity; the other entries are zeros; all rows are different):

$$
\mathbf{f}_{\mathrm{c}, \mathrm{a}}=\mathbf{P}_{\mathrm{a}} \mathbf{f}_{\mathrm{c}} ; \quad \mathbf{f}_{\mathrm{c}, \mathrm{d}}=\mathbf{P}_{\mathrm{d}} \mathbf{f}_{\mathrm{c}}
$$

The vector $\mathbf{f}_{\mathrm{c}}$ is nonzero due to the uncertainties in the parameters and the calculational model itself. We combine the uncertainties of the parameters into the vector $\mathbf{d}_{c}$. The model uncertainties are organized in the vector $m_{c}$. If the uncertainties are small, then $f_{c}$ has a linear dependence on $d_{c}$; the model uncertainty is summed with the uncertainty propagated from the parameters:

$$
\mathbf{f}_{\mathbf{c}}=\mathbf{A d}_{\mathbf{c}}+\mathbf{m}_{\mathrm{c}}
$$

where $\mathbf{A}$ is a sensitivity matrix of the calculational results to the parameters. The linear model is an inherent limitation of the formalism, which is based on uncertainty description by covariances (Fröhner, 1994). The vector $y$ of differences between the calculational and the experimental values of the auxiliary quantities equals the differences in the uncertainties:

$$
\mathbf{y}=\mathbf{f}_{\mathrm{c}, \mathrm{a}}-\mathbf{f}_{\mathrm{c}, \mathrm{a}}=\mathbf{P}_{\mathrm{a}} \mathbf{f}_{\mathrm{c}}-\mathbf{f}_{\mathrm{e}, \mathrm{a}}
$$

Multiplying the vector $y$ with its transpose, averaging and accounting for the independence of the calculation and the experiment we get the covariance matrix:

$$
\left\langle\mathbf{y} \mathbf{y}^{\mathrm{T}}\right\rangle=\mathbf{P}_{\mathrm{a}}\left\langle\mathbf{f}_{\mathrm{c}} \mathbf{f}_{\mathrm{c}}^{\mathrm{T}}\right\rangle \mathbf{P}_{\mathrm{a}}^{\mathrm{T}}+\left\langle\mathbf{f}_{\mathrm{e}, \mathrm{a}} \mathbf{f}_{\mathrm{e}, \mathrm{a}}^{\mathrm{T}}\right\rangle
$$

Utilizing equation (11) we get the expression for the covariances of the calculational uncertainties: 


$$
\left\langle\mathbf{f}_{c} \mathbf{f}_{c}^{\top}\right\rangle=\mathbf{A}\left\langle\mathbf{d}_{c} \mathbf{d}_{c}^{\top}\right\rangle \mathbf{A}^{\mathrm{T}}+\left\langle\mathbf{m}_{c} \mathbf{m}_{c}^{\top}\right\rangle
$$

where the independence of the model and parameters is taken into consideration.

The correlation matrices for the vectors $\mathbf{d}_{\mathrm{c}}, \mathbf{m}_{\mathrm{c}}, \mathbf{f}_{\mathrm{c}}, \mathbf{f}_{\mathrm{e}, \mathrm{a}}$ and vector $\mathbf{y}$ are composed in a similar way:

$$
\begin{aligned}
\left\langle\mathbf{d}_{c} \mathbf{y}^{\top}\right\rangle & =\left\langle\mathbf{d}_{c} \mathbf{d}_{c}^{\mathrm{T}}\right\rangle \mathbf{A}^{\mathrm{T}} \mathbf{P}_{\mathrm{a}}^{\mathrm{T}} \\
\left\langle\mathbf{m}_{\mathrm{c}} \mathbf{y}^{\mathrm{T}}\right\rangle & =\left\langle\mathbf{m}_{\mathrm{c}} \mathbf{m}_{\mathrm{c}}^{\mathrm{T}}\right\rangle \mathbf{P}_{\mathrm{a}}^{\mathrm{T}} ; \\
\left\langle\mathbf{f}_{\mathrm{c}} \mathbf{y}^{\mathrm{T}}\right\rangle & =\left\langle\mathbf{f}_{\mathrm{c}} \mathbf{f}_{\mathrm{c}}^{\mathrm{T}}\right\rangle \mathbf{P}_{\mathrm{a}}^{\mathrm{T}} \\
\left\langle\mathbf{f}_{\mathrm{e}, \mathrm{a}} \mathbf{y}^{\mathrm{T}}\right\rangle & =-\left\langle\mathbf{f}_{\mathrm{e}, \mathrm{a}} \mathbf{f}_{\mathrm{e}, \mathrm{a}}^{\mathrm{T}}\right\rangle
\end{aligned}
$$

When both the calculation and the experiment are performed and we hold the observed value $\mathbf{Y}$ of the vector $\mathbf{y}$, equations (7) and (8) can be employed for the description of this posterior case. For the vectors $d_{c}$, $\mathbf{m}_{c}, \mathbf{f}_{\mathrm{c}}, \mathbf{f}_{\mathrm{c}, \mathrm{a}}$ we obtain the posterior means

$$
\begin{gathered}
\left\langle\mathbf{d}_{\mathrm{c}}\right\rangle_{\mathrm{o}}=\left\langle\mathbf{d}_{\mathrm{c}} \mathbf{y}^{\mathrm{T}}\right\rangle\left\langle\mathbf{y} \mathbf{y}^{\mathrm{T}}\right\rangle^{-1} \mathbf{Y}=\left\langle\mathbf{d}_{\mathrm{c}} \mathbf{d}_{\mathrm{c}}^{\mathrm{T}}\right\rangle \mathbf{A}^{\mathrm{T}}\left\langle\mathbf{y} \mathbf{y}^{\mathrm{T}}\right\rangle^{-1} \mathbf{Y} \\
\left\langle\mathbf{m}_{\mathrm{c}}\right\rangle_{\mathrm{o}}=\left\langle\mathbf{m}_{\mathrm{c}} \mathbf{y}^{\mathrm{T}}\right\rangle\left\langle\mathbf{y} \mathbf{y}^{\mathrm{T}}\right\rangle^{-1} \mathbf{Y}=\left\langle\mathbf{m}_{\mathrm{c}} \mathbf{m}_{\mathrm{c}}^{\mathrm{T}}\right\rangle \mathbf{P}_{\mathrm{a}}^{\mathrm{T}}\left\langle\mathbf{y} \mathbf{y}^{\mathrm{T}}\right\rangle^{-1} \mathbf{Y} \\
\left\langle\mathbf{f}_{\mathrm{c}}\right\rangle_{\mathrm{o}}=\left\langle\mathbf{f}_{\mathrm{c}} \mathbf{y}^{\mathrm{T}}\right\rangle\left\langle\mathbf{y} \mathbf{y}^{\mathrm{T}}\right\rangle^{-1} \mathbf{Y}=\left\langle\mathbf{f}_{\mathrm{c}} \mathbf{f}_{\mathrm{c}}^{\mathrm{T}}\right\rangle \mathbf{P}_{\mathrm{a}}^{\mathrm{T}}\left\langle\mathbf{y} \mathbf{y}^{\mathrm{T}}\right\rangle^{-1} \mathbf{Y} \\
\left.\left\langle\mathbf{f}_{\mathrm{e}, \mathrm{a}}\right\rangle_{\mathrm{o}}=\left\langle\mathbf{f}_{\mathrm{e}, \mathrm{a}} \mathbf{y}^{\mathrm{T}}\right\rangle \mathbf{y} \mathbf{y}^{\mathrm{T}}\right\rangle^{-1} \mathbf{Y}=-\left\langle\mathbf{f}_{\mathrm{e}, \mathrm{a}} \mathbf{f}_{\mathrm{e}, \mathrm{a}}^{\mathrm{T}}\right\rangle\left\langle\mathbf{y} \mathbf{y}^{\mathrm{T}}\right\rangle^{-1} \mathbf{Y}
\end{gathered}
$$

and the posterior covariance matrices:

$$
\begin{aligned}
\left\langle\mathbf{d}_{\mathrm{c}} \mathbf{d}_{\mathrm{c}}^{\mathrm{T}}\right\rangle_{\mathrm{o}} & =\left[\mathbf{I}-\left\langle\mathbf{d}_{\mathrm{c}} \mathbf{d}_{\mathrm{c}}^{\mathrm{T}}\right\rangle \mathbf{A}^{\mathrm{T}} \mathbf{P}_{\mathrm{a}}^{\mathrm{T}}\left\langle\mathbf{y} \mathbf{y}^{\mathrm{T}}\right\rangle^{-1} \mathbf{P}_{\mathrm{a}} \mathbf{A}\right]\left\langle\mathbf{d}_{\mathrm{c}} \mathbf{d}_{\mathrm{c}}^{\mathrm{T}}\right\rangle ; \\
\left\langle\mathbf{m}_{\mathrm{c}} \mathbf{m}_{\mathrm{c}}^{\mathrm{T}}\right\rangle_{\mathrm{o}} & =\left[\mathbf{I}-\left\langle\mathbf{m}_{\mathrm{c}} \mathbf{m}_{\mathrm{c}}^{\mathrm{T}}\right\rangle \mathbf{P}_{\mathrm{a}}^{\mathrm{T}}\left\langle\mathbf{y} \mathbf{y}^{\mathrm{T}}\right\rangle^{-1} \mathbf{P}_{\mathrm{a}}\right]\left\langle\mathbf{m}_{\mathrm{c}} \mathbf{m}_{\mathrm{c}}^{\mathrm{T}}\right\rangle ; \\
\left\langle\mathbf{f}_{\mathrm{c}} \mathbf{f}_{\mathrm{c}}^{\mathrm{T}}\right\rangle_{\mathrm{o}} & =\left[\mathbf{I}-\left\langle\mathbf{f}_{\mathrm{c}} \mathbf{f}_{\mathrm{c}}^{\mathrm{T}}\right\rangle \mathbf{P}_{\mathrm{a}}^{\mathrm{T}}\left\langle\mathbf{y} \mathbf{y}^{\mathrm{T}}\right\rangle^{-1} \mathbf{P}_{\mathrm{a}}\right]\left\langle\mathbf{f}_{\mathrm{c}} \mathbf{f}_{\mathrm{c}}^{\mathrm{T}}\right\rangle \\
\left\langle\mathbf{f}_{\mathrm{e}, \mathbf{a}} \mathbf{f}_{\mathrm{c}, \mathrm{a}}^{\mathrm{T}}\right\rangle_{\mathrm{o}} & =\left[\mathbf{I}-\left\langle\mathbf{f}_{\mathrm{e}, \mathbf{a}} \mathbf{f}_{\mathrm{c}, \mathrm{a}}^{\mathrm{T}}\right\rangle\left\langle\mathbf{y} \mathbf{y}^{\mathrm{T}}\right\rangle^{-1}\right]\left\langle\mathbf{f}_{\mathrm{e}, \mathbf{a}} \mathbf{f}_{\mathrm{c}, \mathrm{a}}^{\mathrm{T}}\right\rangle
\end{aligned}
$$

where I is a unity matrix of proper dimensions.

The difference with Section 2 is, that in the present analysis we deal with only two independent informational sources, allowing, however, deeper insight into their nature by distinguishing explicitly between the auxiliary and desired quantities, between the model uncertainties and uncertainties propagated from the parameters.

In spite of the seeming complexity of the cited formulas, the annotation is quite modest. Indeed, before the confluence the vector $y$ is defined and interpreted as the error of the calculation diminished by the error of the experiment. This is a random vector-it has a zero mean, but a nontrivial covariance. Because there is no correlation between the calculation and the experiment before the confluence, the covariance matrices of the corresponding errors are merely summed up, in agreement with equation (13).

The total uncertainty of the informational system does not change

$$
\left\langle\mathbf{y} \mathbf{y}^{\mathrm{T}}\right\rangle_{0}=\left\langle\mathbf{y} \mathbf{y}^{\mathrm{T}}\right\rangle
$$

and the observed value $Y$ is preserved for the mean of the posterior $y_{0}$, but $y_{0}$ is now a systematic bias, uncovered by the confluence. The mean $\mathbf{Y}$ must be covered by two partial-calculational and experimental-posterior means, so that after the confluence it is appropriate to interpret $\mathbf{y}_{0}$ as the "total" bias - the sum of $\mathbf{P}_{\mathrm{a}}\left\langle\mathbf{f}_{\mathrm{c}}\right\rangle_{\mathrm{o}}$ and $-\left\langle\mathbf{f}_{\mathrm{e}, \mathrm{a}}\right\rangle_{\mathrm{o}}$. The proportion in which the total mean $\mathbf{Y}$ is parceled to set apart the 
partial biases in the posterior case is determined by prior knowledge - by the relative contributions of the prior partial uncertainty covariances $\mathbf{P}_{\mathrm{a}}\left\langle\mathbf{f}_{\mathrm{c}} \mathbf{f}_{\mathrm{c}}^{\mathrm{T}}\right\rangle \mathbf{P}_{\mathrm{a}}^{\mathrm{T}}$ and $\left\langle f_{\mathrm{e}, \mathrm{a}} \mathbf{f}_{\mathrm{e}, \mathrm{a}}^{\mathrm{T}}\right\rangle$ into the prior total uncertainty covariance $\left\langle\mathbf{y y y}^{\mathrm{T}}\right\rangle$ in correspondence with equation (13). This is expressed by equations (21) and (22). Judging the nature of the calculational uncertainty leads to equations (19) and (20), which acknowledge the similar interpretation. The posterior covariances of the partial uncertainties are the weighted prior ones. The weights are the unit operators, decreased by the relative contributions of the prior covariances of the partial uncertainty into the prior covariance of the total uncertainty.

\section{APPLICATION OF THE CONFLUENCE TECHNIQUE TO THE DETERMINATION OF POWER DISTRIBUTION AND BURNUP OF THE HOR REACTOR}

HOR (Hoger Onderwijs Reactor) is a $2 \mathrm{MWth}$ pool-type research reactor situated at the Interfaculty Reactor Institute in Delft, The Netherlands. It contains highly enriched MTR-type fuel elements.

The reactor is controlled by means of four $\mathrm{B}_{4} \mathrm{C}$ shim-safety rods. The core grid plate has 42 positions, normally loaded with fuel elements including control ones and several reflector elements, containing $\mathrm{BeO}$ or Be-metal. In some positions there are experimental facilities.

The reactor is operated continuously 5 days a week. The licensed excess-reactivity is $6 \%$. This requires replacement of a few elements and reshuffling at a three-month interval. Sample configurations and corresponding replacement of the elements for two subsequent cycles used in the calculations are presented in Fig. 1.

After completion of the reshuffling operation the power distribution is calculated. This distribution is used in burnup calculations which are also performed quarterly.

An approach used to determine the power distribution employed the product of the neutron flux density and the fission cross section. Foil activities are measured to determine the flux density distributions and power distributions by the PEP (Brand et al., 1971) code. The code BUPNEW (de Vries et al., 1980), based on approximate relationships between the isotopic composition of the fuel to the total energy produced by fission in each fuel element, is used for burnup determination. Neither broadness and accuracy of available nuclear data nor power of modern computer codes is used in the employed methods.

The confluence procedure supplies a principal possibility of developing a comprehensive informational code system working in a reactor maintenance system. Parameters and calculational results obtained by a code based on some physical reactor model can be corrected using the most recent experimental results. The CONHOR program system (Serov and Hoogenboom, 1993) is developed to apply the confluence procedure for determination of the power distribution and burnup for the HOR reactor and to serve as an instrument of fuel management support. The CITATION (Fowler et al., 1971) diffusion code is used as a main calculational tool for CONHOR power-burnup calculations. CONHOR involves CITATION runs in the calculational flow on all the stages of the calculation, when a reactor calculation is necessary.

Calculations are performed using a two-dimensional approach which has the advantage of significant economy of computing time, especially in long-term burnup determination problems. Three-dimensional advanced nodal codes will be definitely a better choice for commercial analysis and their utilization will already significantly reduce the prior model uncertainty of the calculation. However, we have not striven for choosing the best of such methods, but aimed to test the workability of the confluence technique in applied problems.

Besides averaging, the two-dimensional diffusion model brings an additional model uncertainty caused by emulating control rod positioning. Suggested emulation is based on the CITATION possibilities and involves the use of an internal absorbing zone, on which boundary "gray" absorption conditions are specified and an external absorbing zone, where additional material with high absorption cross section is placed. We take here into account the fact that the control element of the HOR reactor is analogous to a normal fuel element, but with a number of inner fuel plates removed. The former (gray) zone is responsible for substantial absorption and originates due to limited possibilities of the diffusion approach in zones with high absorption. The external absorbing zone is used in an additional material concentration search, which is executed as one of the CITATION options to maintain criticality.

Statistical properties of model uncertainties introduced by this (or any other) core physics modeling can be evaluated by comparing the results of calculations for different reactor states with data obtained by a 

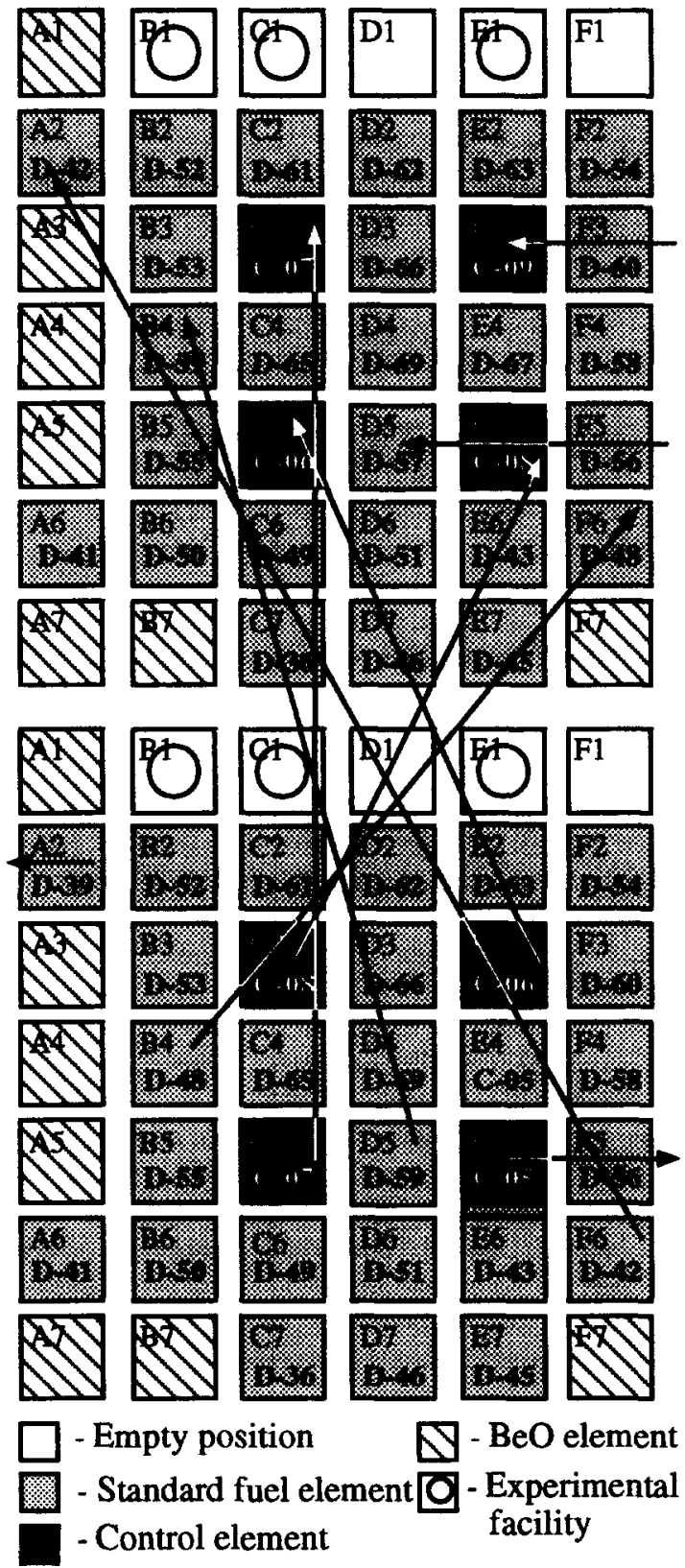

$$
\begin{array}{ll}
\mathrm{A} 1, \mathrm{~B} 1, \ldots & \text { - Grid plate positions } \\
\mathrm{R}-08, \mathrm{D}-39, \ldots & \text { - Element names }
\end{array}
$$

Fig. 1. Configuration and fuel reshuffling; HOR cores 9002 and 9003. Arrows show reshuffling.

Monte-Carlo code, as described in Section 2. Practicing this approach is in its infancy and we have rather used an empirical estimation of the model covariances.

Axially averaged activation rates and fractional power values were estimated to have equal relative standard deviations of both systematic (5\%) and statistical $(5 \%)$ nature. 
The most important data for the CITATION calculation consists of neutron cross sections and nuclide number densities for isotopes. The AMPX-master (Greene et al., 1978) library containing evaluated fine group neutron cross sections data is processed by the BONAMI and NITAWL (Parks et al., 1989) codes for the resonance treatment. A produced AMPX-working library is used by the XSDRNPM (Parks et al., 1989) code and after OCTAGEN (Greene $e t$ al., 1978) postprocessing a CITATION library of neutron cross sections collapsed to five energy groups for six sets of different fuel burnups is obtained. Separate sets for moderator and other materials are formed. Nuclide number densities are specified for steady nuclides. For burning, decaying and accumulating nuclides they are calculated by combination of the XSDRNPM and ORIGEN (Parks et al., 1989) codes. After the postprocessing interpolation these varying nuclide densities are referred to by ${ }^{235} U$ burnup values. The latter were obtained from PEP-BUPNEW calculations for previous cycles which could introduce considerable errors. These errors were estimated by de Vries et al. (1980) as $10 \%$ in the calculation of average burnup of the core. This fact makes us consider ${ }^{235} \mathrm{U}$ number density values as data to be corrected first of all. The systematic and statistical uncertainties are combined allowing zero mean for the ${ }^{235} \mathrm{U}$ density uncertainty. The prior relative standard deviations of this combined uncertainty is estimated to equal $10 \%$ of the burned ${ }^{235} \mathrm{U}$ density for each fuel-containing element in the core. The ${ }^{235} \mathrm{U}$ densities for different elements were presumed uncorrelated. When the CONHOR burnup calculation is used, we should expect a new uncertainty in the burnup determination to be accumulated, which is brought up by the CITATION depletion model. This uncertainty is estimated to be $2 \%$ of the burned ${ }^{235} \mathrm{U}$ density. The corresponding variance is added to the variance at the end of the cycle.

Experimental data consists of gold foil activity measurement results, which are fulfilled in the beginning of every cycle and have been used for PEP-BUPNEW power-burnup determination. Activation rates for all the fuel elements are computed and compared with the calculated ones. The latter values are based on both the flux values at the position of the foil stringers obtained by CITATION and activation cross sections for gold obtained on the same base as above for regular reactor materials. It is assumed that the measurement equipment is properly calibrated so that experimentally obtained activation rates are bias-free. Relative statistical deviations of the measured rates are estimated by Brand et al. (1971) to be $3 \%$.

Input data for CONHOR data is collected in two files. One of them contains general information describing the reactor. It includes information concerning the reactor and models used, geometry description, activation cross sections, statistical data, etc., which can be needed in different stages of the calculation and do not differ from cycle to cycle. The second file combines data relating to a particular style, such as loading of the reactor (correspondence between the positions in the grid and unique element names), measured activity data and operating schedule description. Both files are divided into sections with different types of data, which are accessed sequentially as far as needed. There is a library of cross sections supplied to be used by CITATION and a library of fuel element descriptions, which contains nuclide number densities. Records are available by direct access and referred to by unique element names. CONHOR is responsible for creating automatically CITATION input files in accordance with the purpose of the calculation to be executed.

The general informational flow of CONHOR is presented in Fig. 2. The corresponding calculational flow of the system can be described as follows. CONHOR creates a CITATION input file for a basic static calculation. Such a calculation is implemented, point-wise power density distribution and point- and groupwise flux density distribution prepared by CITATION are used by CONHOR to compute the power and activation rate distributions. Then an adjustment procedure can be requested. It includes direct calculations of sensitivity coefficients by perturbing ${ }^{235} U$ densities in the elements followed by the confluence procedure based on the formalism described in Section 3. Corrected values of the ${ }^{235} U$ are stored in a new library. Afterwards, a burnup calculation based on corrected ${ }^{235} \mathrm{U}$ densities can be performed. Alternatively a burnup calculation can be done excluding adjustment. There is an option to request no burnup calculation.

If burnup is executed, then another library with densities at the end of the cycle is produced. It can be a library based on either corrected or uncorrected ${ }^{235} \mathrm{U}$ densities at the beginning of the cycle, so that all types of comparisons can be made. The library used at the beginning of the cycle includes also data for the elements which will be loaded at the beginning of the next cycle. These data are not used in the calculations, but will be available in the new library at the end of the cycle, so that the latter can directly be used in the calculations of the next cycle.

Long-lasting CITATION calculations, once completed, can be easily excluded from the calculational flow, if there is a need to repeat CONHOR calculations with another set of data not influencing the CITATION output distributions. Both this and CONHOR-restart options are very useful for analysis and optimization. 


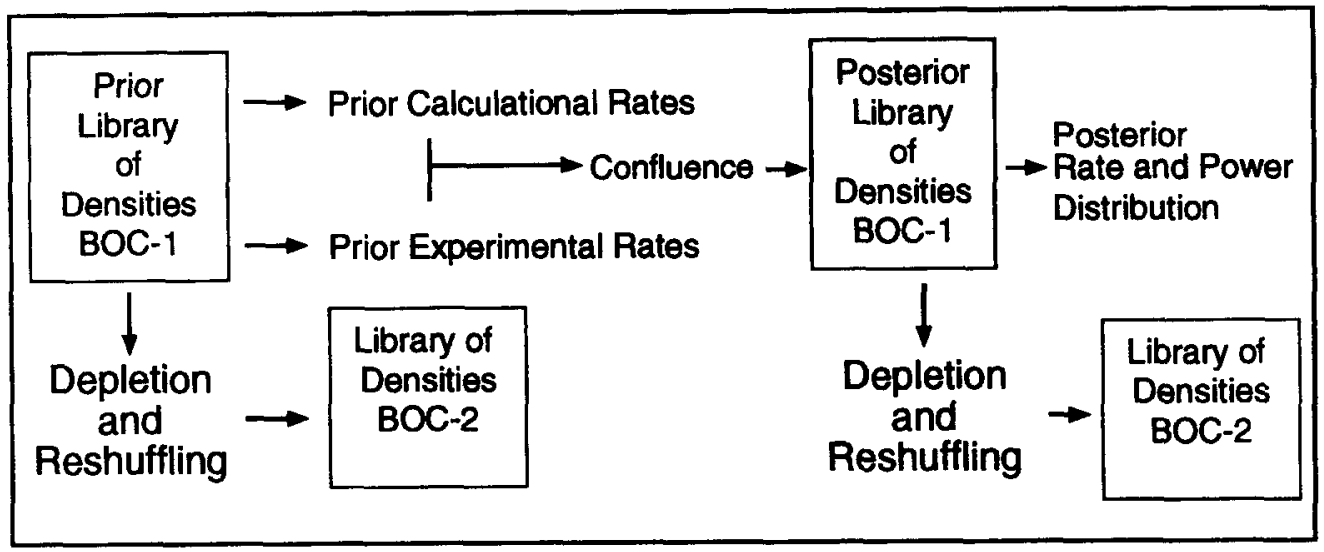

Fig. 2. Informational flow of CONHOR. BOC-1-Beginning of the first studied cycle; BOC-2-beginning of the second studied cycle.

\section{NUMERICAL RESULTS}

For testing calculations a combination of cycles number 9002 and 9003 has been chosen. These are two consecutive cycles the HOR reactor passed through in the second and third quarters of the year 1990 . We refer here again to Fig. 1.

Three different approaches were exercised to test the program and to study the confluence effects. According to the first approach (to be called "A") number densities prepared by PEP-BUPNEW are used for the beginning of cycle (BOC) 9002. The confluence procedure is applied and a new library with the corrected ${ }^{235} \mathrm{U}$ densities is created. This library is used in the burnup calculations for cycle 9002 . At the end of cycle (EOC) the corresponding library is available and used at BOC 9003 . The confluence procedure is applied again and after the reactor calculation is performed, the results are referred to as the results of the $\mathrm{A}$ approach for BOC 9003. In the second approach, "B", the corrections are applied only in BOC 9003 and not for the 9002 cycle, so that the burnup calculation for the first cycle is performed with the uncorrected ${ }^{235} \mathrm{U}$ densities. According to the third approach, "C", no CONHOR calculations are done for the 9002 cycle and the correction procedure is applied directly in the BOC 9003 cycle using the number density library, prepared by means of PEP-BUPNEW for that cycle.

The results of the B-approach, where the CITATION procedure is exercised, but not the PEP-BUPNEW burnup procedure, are expected to be better than the results of the C-approach. In the same way, approach A can be expected to be better than approach B as the ${ }^{235} \mathrm{U}$ densities are less trustful in the BOC 9002 cycle in the latter case. Approach A seems to lead to the most reliable results, as two independent sets of experimental data are used in the calculation.

The data for approach $\mathrm{C}$ before the confluence are considered as the prior unconditional reference knowledge. The observed differences between the calculational and the experimental activation rates are mostly lower than the total uncertainty of the system (see Fig. 3).

The posterior rates, obtained according to the three approaches, are shown in Fig. 4. The total uncertainty of the system has been reduced by roughly $35 \%$ on average for the activation rate in every individual position. Relative standard deviations for all posterior rates are about $2.6 \%$.

The reasons for the existing differences between the results of the approaches are more deterministic by nature than probabilistic. Indeed, starting with the same set of densities in BOC 9002 we follow different tracks, which are strongly correlated with each other. First of all, we use the same calculational facilities - CONHOR including CITATION. Secondly, the measured foil activities for the cycles 9002 and 9003 are the data for the same physical elements (except a few reshuffled), taken just at different moments in their history. The relative differences in results account for at most about $5 \%$ of the posterior relative standard deviations, which supports the idea of the CONHOR system stability. Another observation is that the average relative correction to the unbiased experimental activation rates is about $5 \%$ of the average posterior relative standard deviation, so that posterior rates look unbiased as well. 


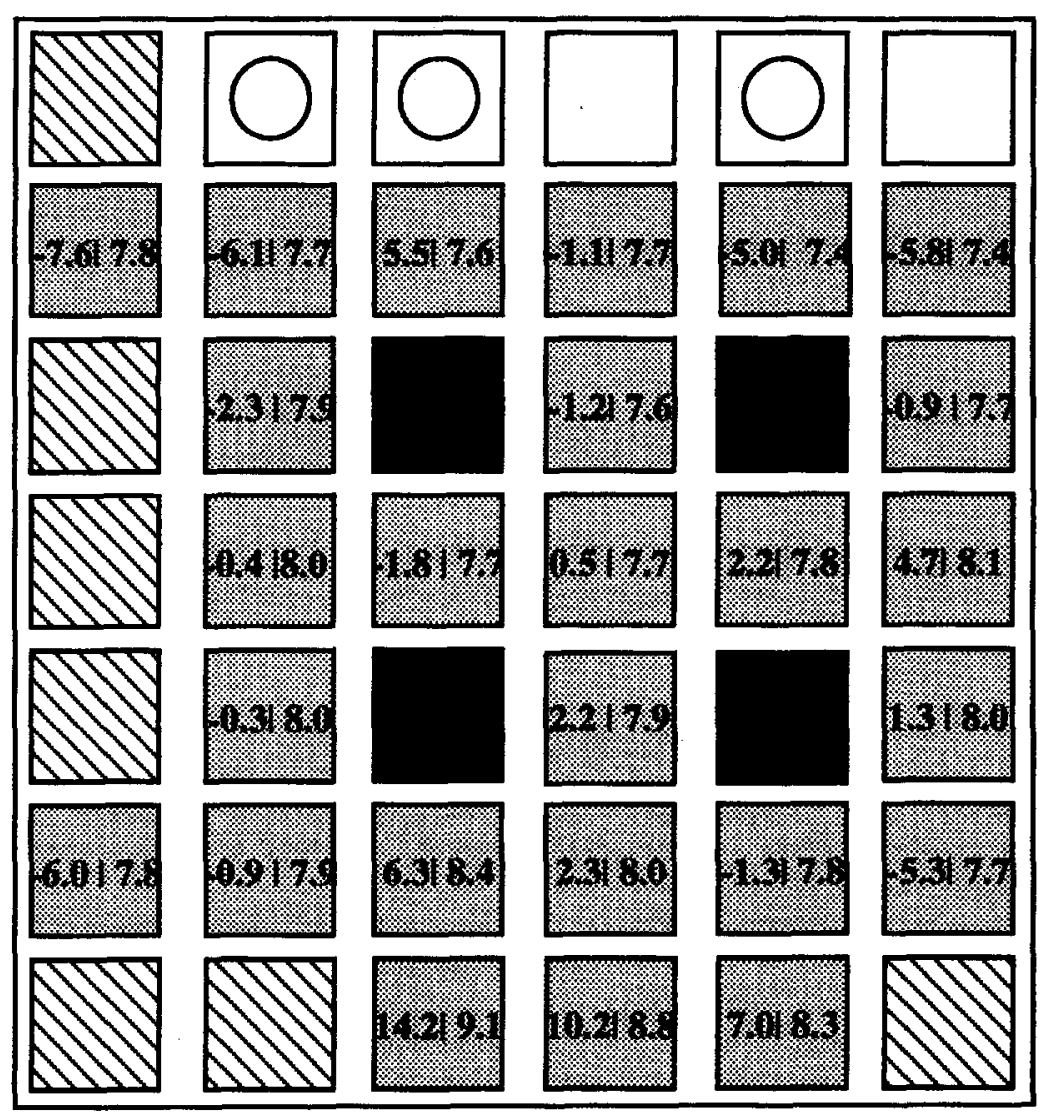
Rates (\%) | Prior Total Relative Standard Deviation of the Activation Rate (\%).

Fig. 3. Comparison of calculational and experimental activation rates. Approach C. BOC 9003.

The observed differences between the calculational and experimental rates are covered by three partial contributors - corrections to the experiment, model and ${ }^{235} U$ densities. In Fig. 5 the relative contributions to the activation rate corrections are shown. The famous cancellation of errors can be explicitly observed at several grid positions. There are two types of such cancellation - either it is not strictly exhibited (1-2\% of the total applied correction), or the total observed correction itself is small (less than $1 \%$ of the experimental rate in the considered grid position). This fact justifies the necessity of explicit uncertainty treatment. It is interesting to note that because the covariance matrix $\mathbf{A}\left\langle\mathbf{d}_{c} \mathbf{d}_{c}^{\mathrm{T}}\right\rangle \mathbf{A}^{\mathrm{T}}$ of the propagated uncertainties from the ${ }^{235} \mathrm{U}$ density is not diagonal, the contributions to the total correction for the specific activation rate are not limited by only local uncertainties in the corresponding grid position.

Because of the clear connection between ${ }^{235} \mathrm{U}$ number densities and the desired burnup data, the adjustment of the latter is straightforward and exhibits the same features. Both the posterior differences between the burnup values for the three approaches (see Fig. 6) and their standard deviations (see Fig. 7) are smaller for every fuel element than the corresponding prior data. The average differences between the ${ }^{235} \mathrm{U}$ densities for the three approaches reduced by more than 10 times. The ${ }^{235} U$ densities obtained in the framework of approach $\mathrm{A}$ show the lowest uncertainty. It is important to note that the corrections applied to the ${ }^{235} \mathrm{U}$ densities combine both the systematic and the statistical components of the uncertainty. The average tendency 

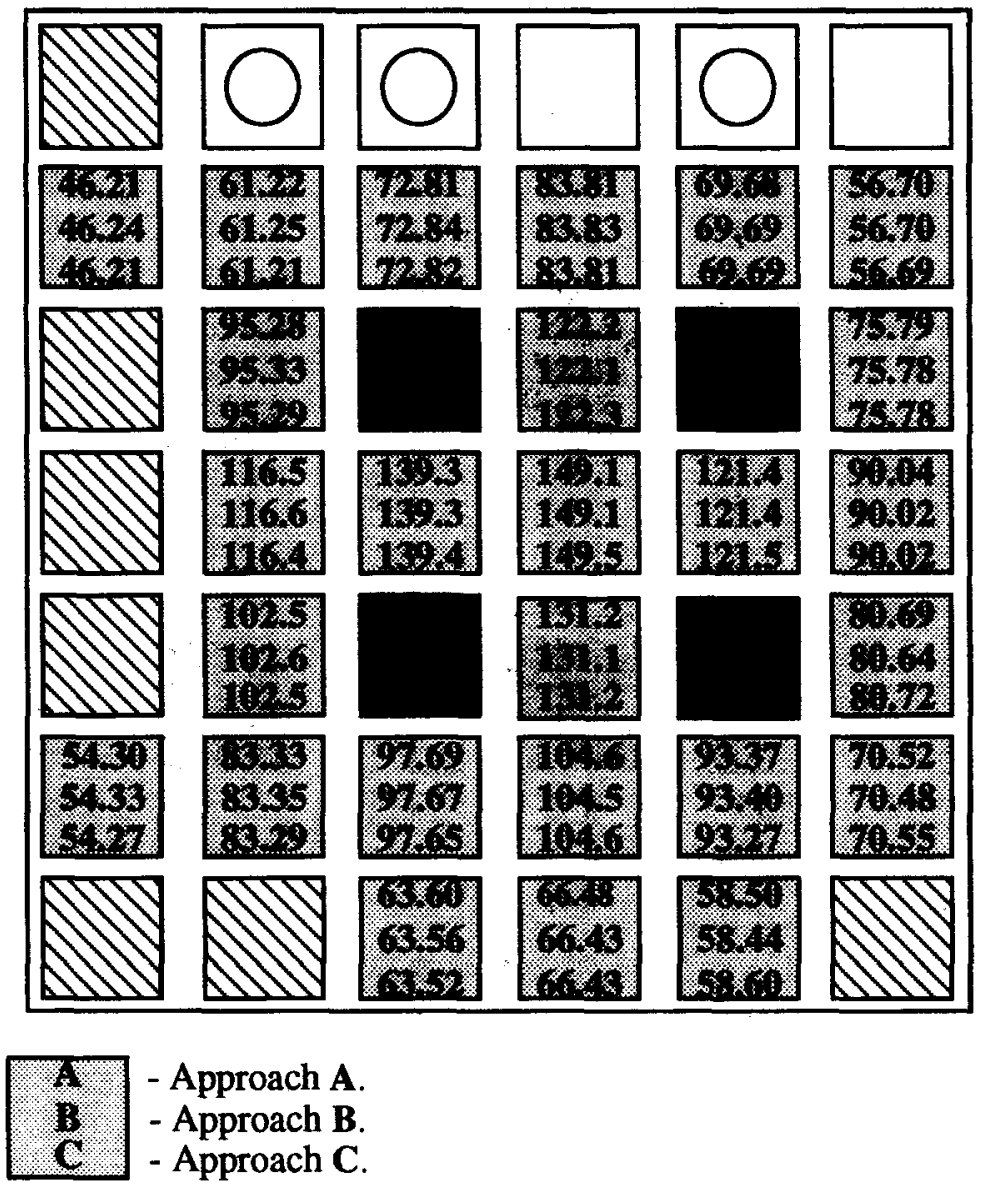

Fig. 4. Comparison of posterior average activation rates $\left(10^{-13} \mathrm{~s}^{-1}\right)$. BOC 9003 .

is to decrease the density, so that the corrections to them seem to correlate with each other. Owing to the Gaussian treatment of the systematic uncertainty, the confluence allows us to detect the existence of the nonzero systematic component in the prior ${ }^{235} U$ uncertainties and even to determine their numerical values.

The posterior values of the power distribution for different approaches differ within the limits of the posterior deviations and the approach $\mathrm{A}$, which has the smallest deviations, is considered to give the best prediction. The recommended power distribution together with the prior one is presented in Fig. 8 .

\section{CONCLUSIONS}

The technique for statistical confluence of any number of possibly correlated informational sources used in reactor analysis can be applied to improve the different estimates of physical quantities. The cited formulas of the method are based on the Bayesian conditioning theorem and pretend to be general enough to provide the mathematical description of the linear adjustment procedures in different types of applications. The confluence technique provides a framework for a comprehensive reactor data system, which can utilize all the available informational sources to offer optimal estimates of the required quantities and their uncertainties.

The possible approach to the evaluation of the necessary explicit knowledge of the systematic and statistical model uncertainties of the prior data is described. The practical utilization of the approach for such evaluation would require long-term Monte-Carlo calculations of the entire core to evaluate local functionals together with their variances and coefficients of mutual correlation, which is not feasible at the moment. The progress 


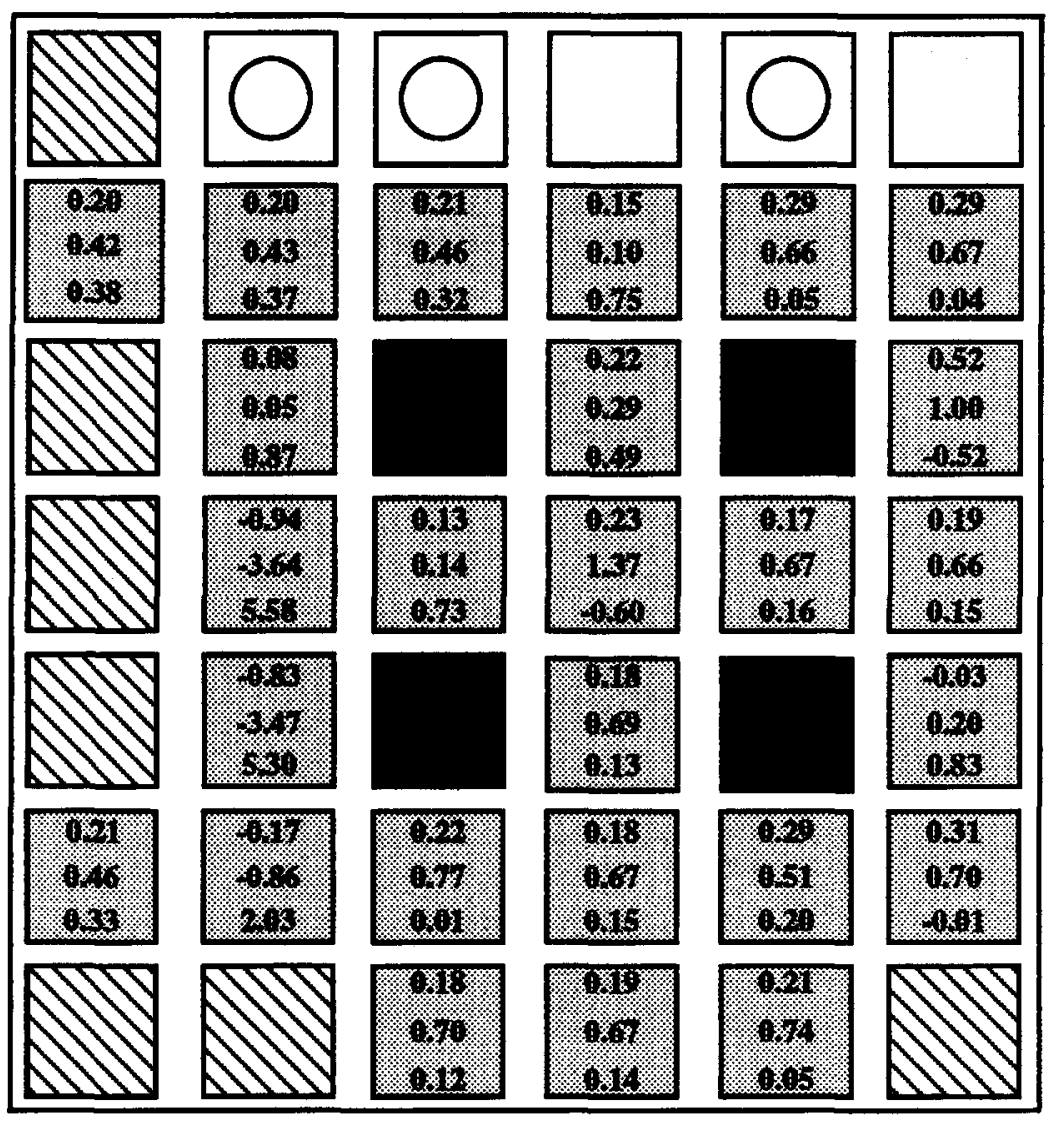

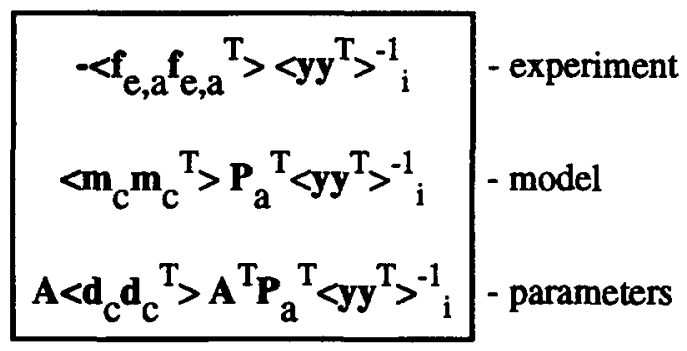

Fig. 5. Comparison of relative partial contributions to the total correction to the activation rates. Approach C. BOC 9003.

is likely to be achieved, if correlation between the local functionals can be neglected and an accurate deterministic code, which is able to evaluate the variances, can be exploited instead of Monte-Carlo.

Another approach is to simplify the evaluation of uncertainty covariances, relying on common sense and expert knowledge. The reliability of the prior evaluation can be judged to a certain extent by the confluence results. Still, the problem of the results' sensitivity to approximations in evaluation of prior statistical properties is as much of scientific interest as of importance.

To avoid the known problem of prior biases, the Gaussian distribution is used for their description. This is the correct procedure (Fröhner, 1994), which handles the troublesome problem of unknown prior systematic uncertainties. Together with confluence it allows us to adjust the data for both systematic and statistical uncertainties simultaneously and in some situations evaluate the magnitudes of them separately.

By means of the CONHOR code system the confluence approach was successfully applied to adjust the 

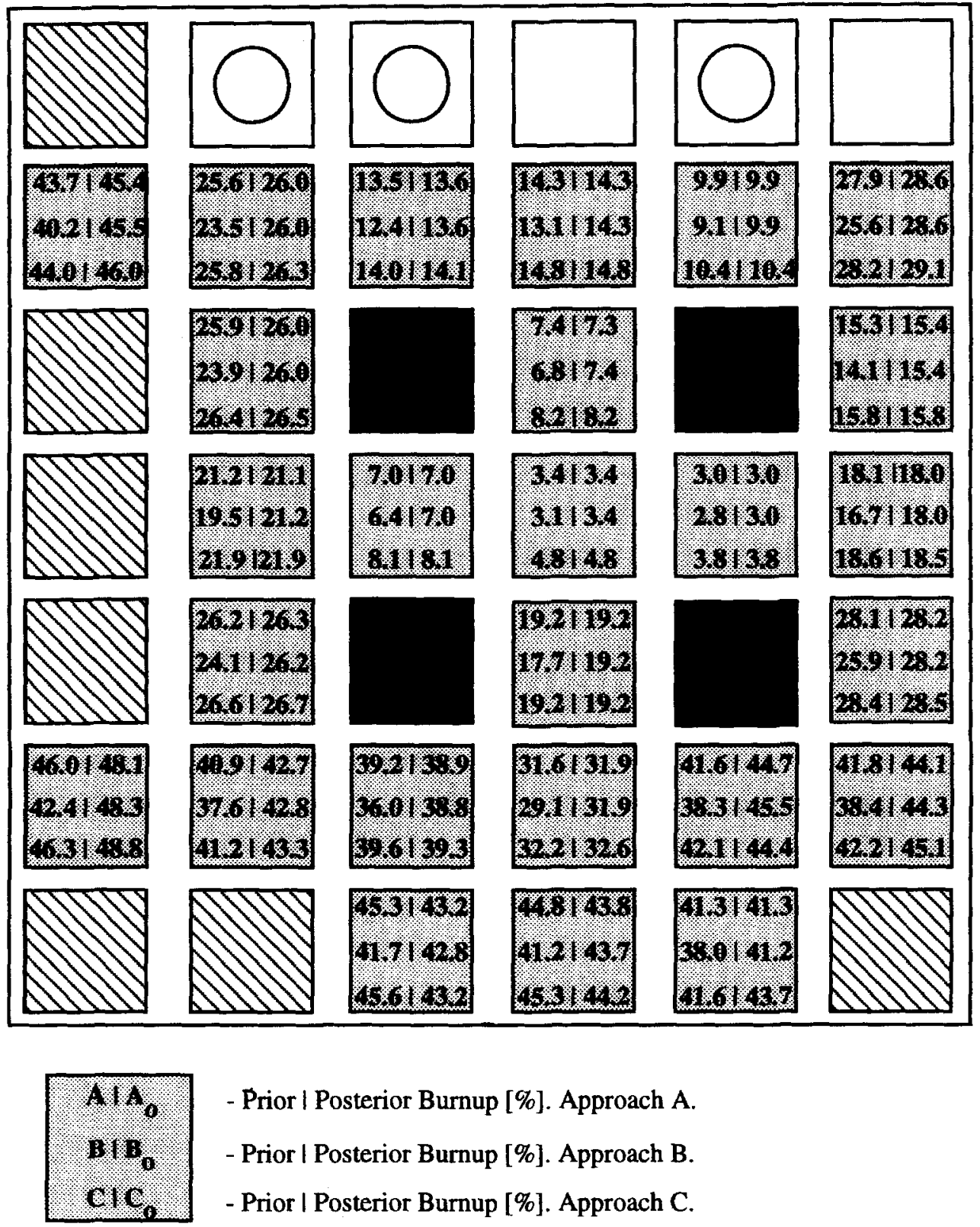

Fig. 6. Adjustment of fuel burnup. BOC 9003.

power and burnup distributions of the HOR reactor. Foil activation rates, power distributions, ${ }^{235} U$ number densities and burnups were corrected within their variances, which are composed to describe the systematic and statistical uncertainty simultaneously. The posterior variances are lower than the prior ones for all the corrected knowledge. A systematic bias of the prior ${ }^{235} \mathrm{U}$ uncertainties reveals itself after the confluence. The use of three different approaches shows the highest efficiency of the one, where the confluence is exercised in the beginning of every cycle, with the burnup calculations based on the improved ${ }^{235} \mathrm{U}$ number densities. 


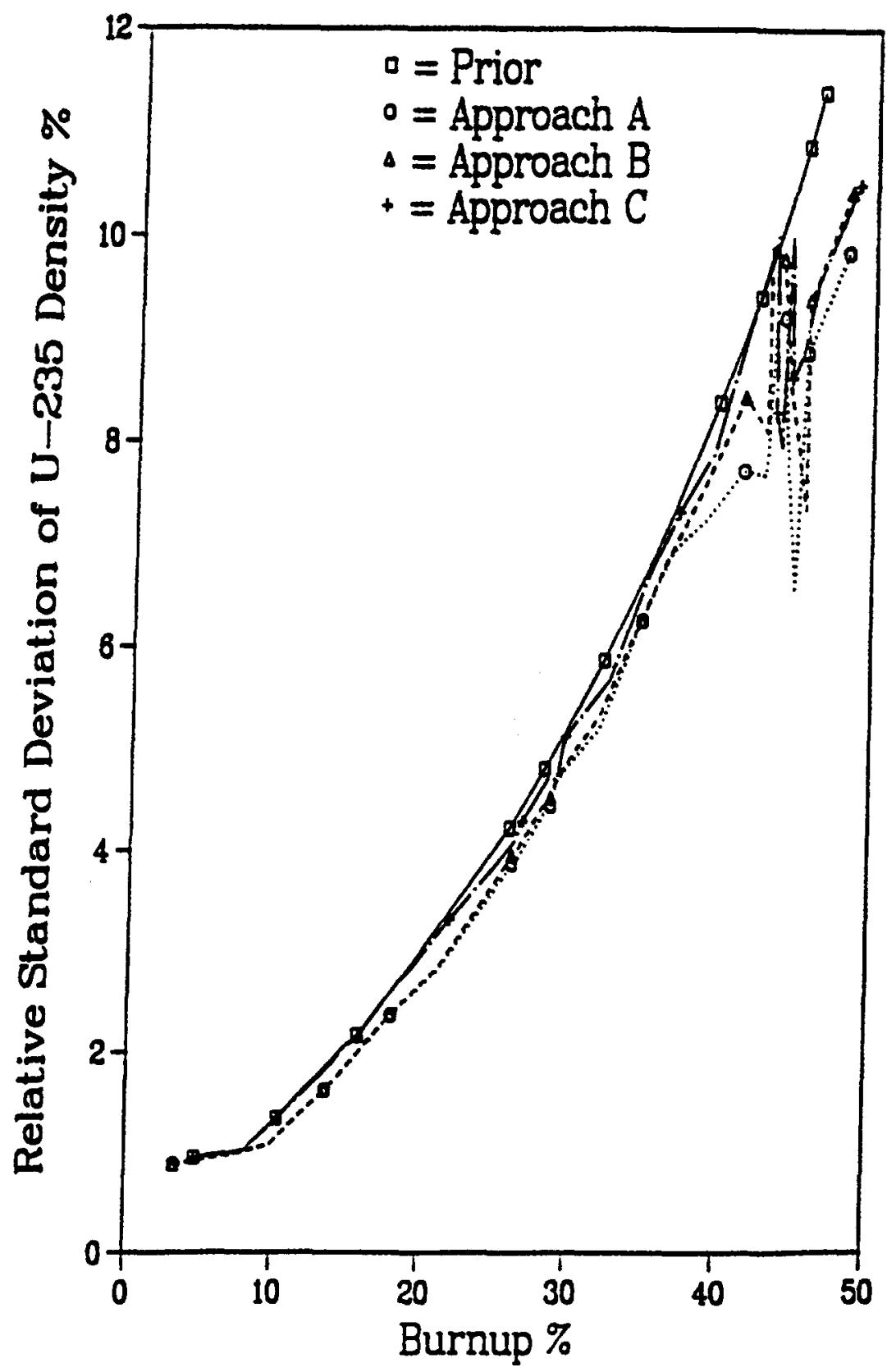

Fig. 7. Improvement of the ${ }^{235} \mathrm{U}$ number density accuracy.

From another side the differences in magnitudes are small enough not to conflict with the conviction of system stability.

The confluence technique proved to be useful for small reactors and the CONHOR system is expected to become a routine instrument of the HOR reactor fuel management support. Future developments will include application of the confluence technique to real power reactors. 

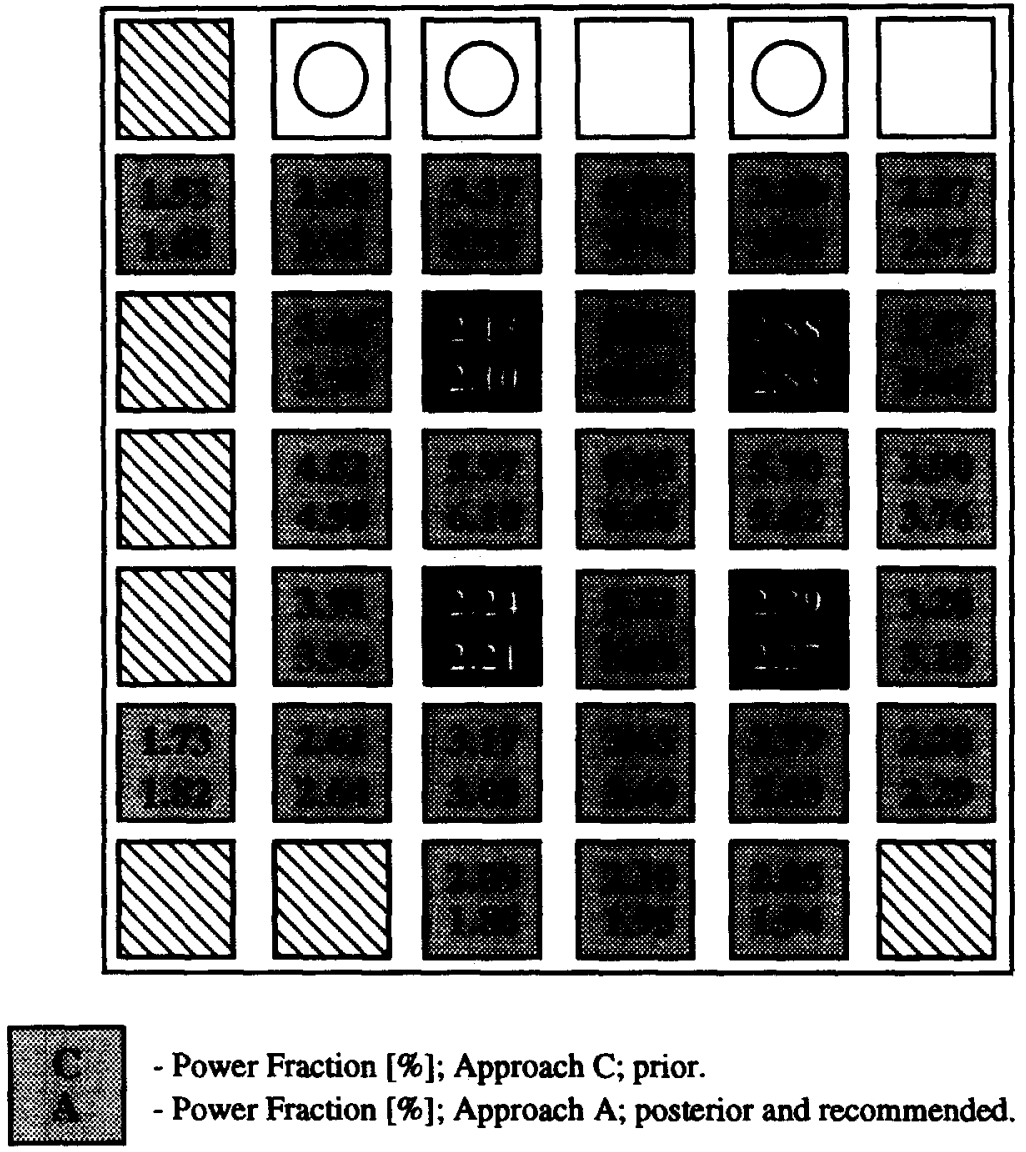

- Power Fraction [\%]; Approach C; prior.

- Power Fraction [\%]; Approach A; posterior and recommended.

Fig. 8. Adjustment of fractional power distribution. BOC 9003.

\section{REFERENCES}

Brand P., van Dam H., de Vries J. W. et al. (1971) Flux-, Power- and Burnup Determination for Pool-Type Reactors, IRI130/131-71-01. Interuniversitair Reactor Institute, Delft.

Bryson J. W., Lee J. C. and Hassberger J. A. (1993) Nucl. Sci. Eng. 114, 238.

Fowler T. B., Vondy D. R. and Cunningham G. W. (1971) CITATION-Nuclear Reactor Analysis Code, TM-2496, Rev. 2. Oak Ridge National Laboratory, Reactor Division.

Fröhner F. H. (1994). Proc. Int. Conf. Reactor Physics and Reactor Computations, Tel-Aviv, Israel, 23-26 January 1994, p. 287. Israel Nuclear Society, European Nuclear Society.

Greene N. M., Licius J. L., Petrie L. M., Ford W. E. et al. (1978) AMPX-A Modular System to Generate Coupled Multigroup Neutron-Gamma Cross Sections from ENDF/B, PSR63/AMPX-II. Oak Ridge National Laboratory, Radiation Shielding Information Center.

Janes E. T. (1983) Papers on Probability, Statistics and Statistical Physics (Edited by R. D. Rozenkrantz). Reidel, Dordrecht. Koroluk B. S. (1985) Reference Book on Probability Theory and Mathematical Statistics, p. 138. Science, Moscow.

Lee P. M. (1989) Bayesian Statistics: An Introduction. Oxford Univ. Press, New York.

Parks C. V., Landers N. F. et al. (1989) SCALE-4-A Modular Code System for Performing Standardized Computer Analyses for Licensing Evaluation, CCC-545. Oak Ridge National Laboratory, Radiation Shielding Information Center.

Perel R. L., Wagschal J. J. and Yeivin Y. (1994) Proc. Int. Conf. Reactor Physics and Reactor Computations, Tel-Aviv, Israel, 23-26 January 1994, p. 15. Israel Nuclear Society, European Nuclear Society.

Serov I. V. and Hoogenboom J. E. (1993) CONHOR Code System for Determination of Power Distribution and Burnup for the HOR Reactor, IRI-131-93-008. Interfacultair Reactor Institute, Delft. 
de Vries J. W., de Leege P. and Stiger B. J. (1980) BUPNEW-Program for the HOR-Reactor Fuel Consumption Bookkeeping, IRI-INT-80-RBG. Interuniversitair Reactor Institute, Delft.

\section{APPENDIX}

\section{Conditional Multidimensional Normal Distribution}

Let a statistical vector $\xi$ have a regular Gaussian distribution with a mean $\langle\xi\rangle$ and the covariance matrix $\left\langle\xi \xi^{\mathrm{T}}\right\rangle$. Let $\xi$ consist of two subvectors $\xi_{1}$ and $\xi_{2}$ with the corresponding means-subvectors $\left\langle\xi_{1}\right\rangle$ and $\left\langle\xi_{2}\right\rangle$. The covariance matrix can be represented as a block matrix $\left\langle\xi^{\top}\right\rangle=\left[\left\langle\xi_{k} \xi_{i}^{\top}\right\rangle, k=1,2, i=1,2\right]$, where $\left\langle\xi_{k} \xi_{i}^{\top}\right\rangle$ are the correlation matrices of the vectors $\xi_{k}$ and $\xi_{i}$. Under the condition that the subvector $\xi_{2}$ exhibits a value $\xi_{2}=\mathbf{Y}$, the conditional distribution of the subvector $\xi_{1}$ is also normal (Koroluk, 1985) with the mean

$$
\left\langle\xi_{1}\right\rangle_{0}=\left\langle\xi_{1}\right\rangle+\left\langle\xi_{1} \xi_{2}^{\top}\right\rangle\left\langle\xi_{2} \xi_{2}^{\top}\right)^{-1}\left(\mathbf{Y}-\left\langle\xi_{2}\right\rangle\right)
$$

and covariance matrix

$$
\left\langle\xi_{1} \xi_{1}^{\top}\right\rangle_{\mathrm{o}}=\left\langle\xi_{1} \xi_{1}^{\top}\right\rangle-\left\langle\xi_{1} \xi_{2}^{\top}\right\rangle\left\langle\xi_{2} \xi_{2}^{\top}\right)^{-1}\left\langle\xi_{2} \xi_{1}^{\top}\right\rangle .
$$

